\title{
Local Knockdown of ERK2 in the Adult Mouse Brain Via Adeno-Associated Virus-Mediated RNA Interference
}

\author{
Barbara Di Benedetto · Benedikt Wefers • \\ Wolfgang Wurst · Ralf Kühn
}

Published online: 4 December 2008

(C) Humana Press 2008

\begin{abstract}
In recent years RNA interference (RNAi) has become a useful genetic tool to downregulate candidate disease genes for which pharmaceutical inhibitors are not available. In combination with viral vectors to trigger RNAi in the mammalian body, it allows the localized and specific manipulation of the expression of single or multiple genes in vivo. The MAP kinases ERK1 and ERK2 are involved in the transduction of extracellular signals to nuclear effectors. A role for ERKs has been proposed in the adult brain in mediating neuronal functions, as for fear learning in the lateral amygdala. To study the role of ERK in anxiety disorders characterized by disturbed fear learning processes we developed Erk-specific RNAi tools and tested the efficacy of a viral Erk2 vector in the adult mouse brain. We found shRNAs that showed silencing of either both ERK1/2 or only ERK2. In particular, our analysis showed that an Erk2-specific shRNA reduced the activity of this gene at comparable efficiency both in vitro and in vivo. This reagent provides a useful tool to study the
\end{abstract}

Barbara Di Benedetto and Benedikt Wefers contributed equally to this work.

B. D. Benedetto · B. Wefers · W. Wurst · R. Kühn $(\bowtie)$ Institute of Developmental Genetics, Helmholtz Zentrum München, German Research Center for Environment and Health $(\mathrm{GmbH})$, Ingolstädter Landstr. 1, 85764 Neuherberg/Munich, Germany

e-mail: ralf.kuehn@helmholtz-muenchen.de

B. D. Benedetto · W. Wurst

Max Planck Institute of Psychiatry, Molecular Neurogenetics,

Kraepelinstrasse 2-10, 80804 Munich, Germany

W. Wurst · R. Kühn

Department of Biosciences, Technical University Munich, Munich, Germany role of ERK2, for which small molecule inhibitors are not available, in the development of anxiety and other psychiatric disorders.

Keywords RNAi - AAV2 - Erk2 - Amygdala · Mouse

\section{Introduction}

The extracellular signal-regulated kinase/mitogen-activated protein kinase (ERK/MAPK) pathway is a signaling cascade involved in diverse cellular functions such as growth, proliferation, and differentiation [1]. Recently, via pharmacological inhibition of ERKs' upstream activators MEK1 and MEK2, a role for ERK1 and ERK2 was also proposed in mature neurons of the rat lateral amygdala (LA) in fear learning [2]. Several brain disorders, like anxiety disorders, are characterized by disturbed fear learning processing [3]. Therefore, the analysis of the molecular pathways responsible for normal and pathological development of fear learning and the search for clinically effective therapeutic tools are highly relevant. Via the analysis of knockout mice for Erk1 and Erk2, it was suggested that the lack of ERK1 does not impair emotional learning [4], while the partial inactivation of ERK2 can affect long-term fear learning [5]. But the simultaneous and ubiquitous expression of Erk1 and Erk2 genes in many regions of the adult murine CNS [6] suggests that the analysis of single knockout animals for these genes is not sufficient to clarify their specific role, due to their potential redundancy which could mislead data interpretation. Thus, classical pharmacology as well as knockout technologies encounter limitations due to: (i) the lack of pharmacological inhibitors targeting specifically the 
ERKs; (ii) the laborious methods necessary for the generation and analysis of single or multiple knockout mice; (iii) the limited number of region-specific promoter regions to allow restricted loss-of-function studies. The discovery of RNA interference (RNAi) can help in mitigating such limitations, allowing the silencing of the expression of single or multiple genes in an efficient and quick manner [7]. In addition, the possibility of using short interfering RNA (siRNA) [8] or short hairpin RNA (shRNA) [9] to trigger RNAi in cells, in combination with viral vectors to perform somatic genetic manipulation, facilitates the specific loss-of-function analysis in selected brain areas [10]. In particular, adeno-associated virus (AAV) reagents have been successfully used to perform gene transfer in the brain via stereotaxic injection [11, 12], as they show several advantages: (i) adaptable diffusion of the vector at the injection site by using different virus serotypes [13]; (ii) predominant infection of neurons rather than glia cells [14]; (iii) very low integration efficiency $(0.1-0.5 \%)$ in the genome of infected cells [15].

In this study, we show the selection and in vitro validation of shRNAs to target and downregulate both Erk1/2 simultaneously, or specifically Erk2, leaving Erk1 unaffected. The further in vivo analysis of AAV-mediated downregulation of Erk2 confirmed our shRNA as a valid tool for the local inhibition of this gene in neurons of the adult mouse brain. This opens the avenue for further studies to analyze the region-specific contribution of ERK2 to the development of anxiety and other psychiatric disorders.

\section{Materials and Methods}

\section{Selection and Validation of shRNA In Vitro}

A Bluescript plasmid containing the human U6 promoter from pSHAG [16] was opened with BseRI/BamHI and ligated with an Erk2-specific oligonucleotide pair (Erksh4) or an Erk1/2-specific oligonucleotide pair (Erksh6) (for sequences see Fig. 1b). The LacZ-specific shRNA vector has been described previously [17]. All plasmids were grown in DH5 $\alpha$, isolated with QIAgen plasmid Maxiprep columns and the integrity of the promoter and shRNA regions was confirmed by DNA sequencing. Mouse F1 ES cells (IDG3.2) were used for transient transfections. ES cells were grown in DMEM medium (Gibco) containing 12.5\% FCS, 20 mM HEPES pH 7.2, 0.1 mM MEM nonessential amino acids (Gibco, Cat. No.: 11140-035), $0.1 \mathrm{mM}$ 2-mercaptoethanol, and $90 \mu \mathrm{l} \mathrm{LIF}\left(10^{7} \mathrm{U} / \mathrm{ml}\right.$, Chemicon) on gelatine coated culture dishes. About $2 \times 10^{6}$ cells were electroporated with each shRNA plasmid and cultivated for $48 \mathrm{~h}$. Cells were then homogenized

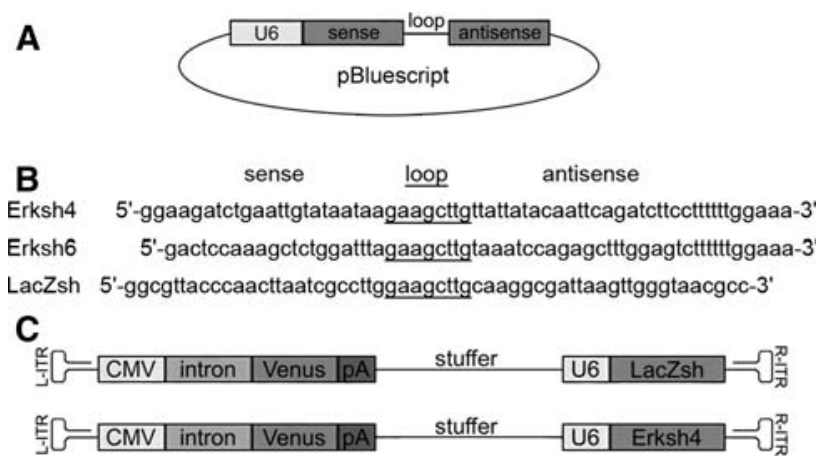

Fig. 1 Schematic drawings of shRNA constructs for in vitro/in vivo studies. a For in vitro studies, the short hairpin RNAs were cloned in pBluescript vector under the control of the U6 promoter (U6). b Sequences of the hairpins tested for their downregulation efficiency via Western blot, after electroporation of the shRNA vector constructs into ES cells. Sequences of the hairpin-loops are underlined. c Schematic representation of the recombinant AAV vector genome AAV-CMV-Venus-U6shRNA. The two expression cassettes for the reporter Venus and the shRNA are separated by a $1.0 \mathrm{~kb}$ stuffer element and the genome is flanked by two $141 \mathrm{bp}$ inverted terminal repeats. ITR inverted terminal repeats; $C M V$ cytomegalovirus promoter; intron: $\beta$-globin intron; Venus: yellow fluorescent protein Venus; pA: human growth hormone polyA signal; stuffer: stuffer element; U6: U6 promoter; LacZsh: control hairpin; Erksh4: hairpin against Erk2

in lysis buffer (2\% SDS, $50 \mathrm{mM}$ Tris pH 6.8, $50 \mathrm{mM}$ DTT, $10 \%$ Glycerol, Protease inhibitor cocktail (Roche, Cat. No.: 11697498001)), and protein concentrations were determined with the BCA test (Pierce) in comparison to BSA as standard; $20 \mu \mathrm{g}$ of each sample were heat denatured, separated on a SDS-PAGE gel, and blotted on PVDF membranes (Pall). Membranes were blocked in 3\% BSA in phosphate-buffered saline with $0.1 \%$ Tween 20 (PBS-T) for $24 \mathrm{~h}$ at $4^{\circ} \mathrm{C}$, incubated with rabbit anti-ERK1/2 (1:1000, Cell Signaling Technology) $1 \mathrm{~h}$ at RT, washed in PBS-T, and incubated with HRP-goat anti-rabbit (1:1000, Jackson ImmunoResearch) $1 \mathrm{~h}$ at RT. After washing, signals were visualized with ECL reagents (Amersham Biosciences) and films were scanned and processed with Adobe Photoshop. For shRNAs that showed performance in Western blot experiments real-time qRT-PCR analysis was performed in addition. Briefly, total RNA was extracted from cells with the Invisorb RNA Kit (Invitek), cDNA synthesis was performed with ABI HighCapacity cDNA reagents and qRT-PCR (Quantace SybrGreen qPCR mix) was run with an ABI 7900HT machine.

\section{Construction of rAAV Vector Plasmid}

Two expression cassettes-one for the yellow fluorescent protein Venus and one either for the shRNA targeting Erk2 or LacZ-were cloned into the pAAV-MCS plasmid (Stratagene) to obtain the expression plasmid CMV-VenusU6shRNA. Venus is a yellow variant of the green 
fluorescent protein GFP, detectable with anti-GFP antibodies [18]. The expression of the reporter gene and the shRNA are driven by the RNA polymerase II-driven CMV and the RNA polymerase III-driven U6 promoter, respectively. The expression cassettes are separated by a nonfunctional stuffer sequence cloned from the $3^{\prime}$ end of the $\beta$ galactosidase gene to obtain an optimal AAV genome size of $4.7 \mathrm{~kb}$.

\section{Production of rAAV Vector}

For vector preparation, ten $100-\mathrm{mm}$ petri dishes of HEK293-derived AAV-293 cells (Stratagene) were transfected by the calcium phosphate technique with $300 \mu \mathrm{g}$ of plasmid DNA (expression plasmid, pHelper and pAAVRC, $100 \mu \mathrm{g}$ each) according to the AAV Helper-Free System manual (Stratagene). After 4 days of incubation, cells were harvested and lysed two times by freeze and thaw technique. Afterward the viral particles were separated from the cell debris by centrifugation.

\section{Purification of rAAV Vector}

Virus suspensions were purified by a single-step column purification [19]; modified protocol) using a heparin-agarose matrix. Viral particles were washed twice with PBS, pH 7.4 and eluted in PBS, pH 7.4 plus $0.5 \mathrm{M} \mathrm{NaCl}$. This eluate was desalted and concentrated with an Amicon Ultra centrifugal filter device (Millipore, $100 \mathrm{~K}$ NMWL).

\section{Characterization of Viral Vectors}

The purity of viral preparations was assessed by electrophoresis on SDS-PAGE gels. Total protein was stained by the Coomassie method. Determination of the genomic titer was achieved by dot blot analysis: a viral sample was degraded with protease $\mathrm{K}$ and loaded on a nitrocellulose membrane. As control, a dilution series of viral cloning plasmids was loaded. For autoradiography, a probe matching the Venus-coding region of the viral genome was used. The in vitro transduction efficiency of viral vectors was determined by incubation of HT1080 cells with a diluted virus preparation. Infected cells expressing the Venus reporter gene were identified by fluorescence microscopy.

Viral Injections and Tissue Preparation for Immunohistochemistry

C57BL/6J adult male mice (Charles River) were anesthetized and stereotaxically injected bilaterally with $1 \mu \mathrm{l} /$ side of AAV preparation into the amygdala. Injections were performed at $0.25 \mu \mathrm{l} / \mathrm{min}$ rate and injectors were left in place for one additional min to allow diffusion of the virus from the tip. Injection coordinates [20] were: $-1.7 \mathrm{~mm}$ anteroposterior, $\pm 3.4 \mathrm{~mm}$ mediolateral, and $-4.0 \mathrm{~mm}$ dorsoventral. After 1 week, mice were killed and perfused with $4 \%$ paraformaldehyde; brains were removed, postfixed overnight, and cryoprotected in 25\% sucrose in PBS. Coronal cryosections $(30 \mu \mathrm{m})$ were cut through the area of the amygdala and kept in cryoprotective solution at $-20^{\circ} \mathrm{C}$ until processed. Experiments involving animals were carried out in accordance with national and institutional guidelines.

Immunofluorescent-Immunohistochemistry (IF-IHC) and in situ Hybridization (ISH)

For the IF-IHC co-localization studies, sections were washed in PBS and incubated with chicken anti-green fluorescent protein antibody (anti-GFP, Aves Lab, 1:500), together with either the specific neuronal marker antiNeuN (mouse, 1:200, Chemicon) or the specific glial marker anti-S100 $\beta$ (mouse, 1:1000, Sigma) in 10\% normal goat serum $/ 0.5 \%$ Triton X-100 in PBS, overnight at $4{ }^{\circ} \mathrm{C}$. On the second day, sections were washed and incubated overnight at $4^{\circ} \mathrm{C}$ with the secondary fluorophore-conjugated antibodies (FITC-anti-chicken, Aves Lab, and TRITC-anti-mouse IgG1, Southern Biotech, both 1:200) and with the nuclear marker DAPI (Sigma). On the third day, sections were washed and mounted onto poly-L-lysinecoated slides for confocal microscopy analysis. For the IFIHC/ISH co-localization studies, the plasmid containing the cDNA for the Erk2 probe was linearized with the appropriate enzyme, purified (PCR purification Kit, QIAgen), and the DIG-labeled probe was prepared by in vitro transcription with DIG RNA Labeling Kit (SP6/T7) (Roche), following manufacturer's instructions. After DNase I (Roche) treatment, the probes were purified by the RNeasy Clean up protocol (QIAgen) and measured in a dot-blot assay, comparing serial dilutions of probe to DIGlabeled control RNA standards. For the ISH procedures, briefly: free-floating sections were rinsed in PBS and endogenous peroxidase quenched with $30 \mathrm{~min}$ incubation in $0.3 \% \mathrm{H}_{2} \mathrm{O}_{2}$ in PBS. Sections were then rinsed in PBS and incubated $15 \mathrm{~min}$ in $5 \times \mathrm{SSC}(0.75 \mathrm{M} \mathrm{NaCl}, 0.75 \mathrm{M}$ Na-citrate). Afterward, they were incubated $1 \mathrm{~h}$ at $58^{\circ} \mathrm{C}$ in pre-hybridization solution $(5 \times \mathrm{SSC}, 50 \%$ formamide, $\mathrm{pH}$ 7.5 with $\mathrm{HCl}, 50 \mu \mathrm{g} / \mathrm{ml}$ salmon sperm) and overnight at $58^{\circ} \mathrm{C}$ in hybridization solution (pre-hybridization solution with $500 \mathrm{ng} / \mathrm{ml}$ of DIG-labeled probe). On the second day, sections were washed $1 \mathrm{~h}$ at $65^{\circ} \mathrm{C}$ in $2 \times \mathrm{SSC}$, then rinsed in PBS, and blocked $30 \mathrm{~min}$ in PBS-BB (PBS with $1 \%$ BSA, $0.2 \%$ powdered skim milk, $0.3 \%$ Triton $\mathrm{X}-100$ ). Afterward, they were incubated $1 \mathrm{~h}$ in HRP-conjugated anti-DIG antibody (Roche), washed in PBS and in PBST 
(0.5\% Triton X-100 in PBS). For amplification of the signal, sections were incubated $20 \mathrm{~min}$ in Tyramid Signal Amplification solution (TSA, Perkin Elmer) (dilution 1:50 in its own diluent solution). They were then rinsed in Maleic acid buffer (MB, 0.1 M Maleic acid, $0.15 \mathrm{NaCl}, \mathrm{pH}$ 7.5 with $\mathrm{NaOH}$ ) and incubated $30 \mathrm{~min}$ in Neutravidin solution (Pierce) (dilution 1:750 in Maleic acid blocking buffer ( $1 \%$ blocking reagent, Roche, in $\mathrm{MB})$ ). After a wash in $\mathrm{MB}$, sections were rinsed in Detection buffer $(0.1 \mathrm{M}$ Tris- $\mathrm{HCl}, \mathrm{pH}$ 9.5; $\left.0.05 \mathrm{M} \mathrm{MgCl}_{2} ; 0.1 \mathrm{M} \mathrm{NaCl}\right)$ and developed in Developing solution $(0.5 \mathrm{mg} / \mathrm{ml} \mathrm{NBT}$, $0.375 \mathrm{mg} / \mathrm{ml}$ BCIP (Roche) in Detection buffer). When a clear signal was visible, sections were washed in PBS and incubated overnight with chicken anti-GFP antibody (as above, 1:500) in 10\% goat serum/0.5\% TX-100 in PBS. After washing in PBS, sections were incubated overnight with the secondary fluorescent-conjugated antibody (FITCanti-chicken, as above, 1:200) in 10\% goat serum $/ 0.5 \%$ TX-100 in PBS. After washing, sections were mounted on
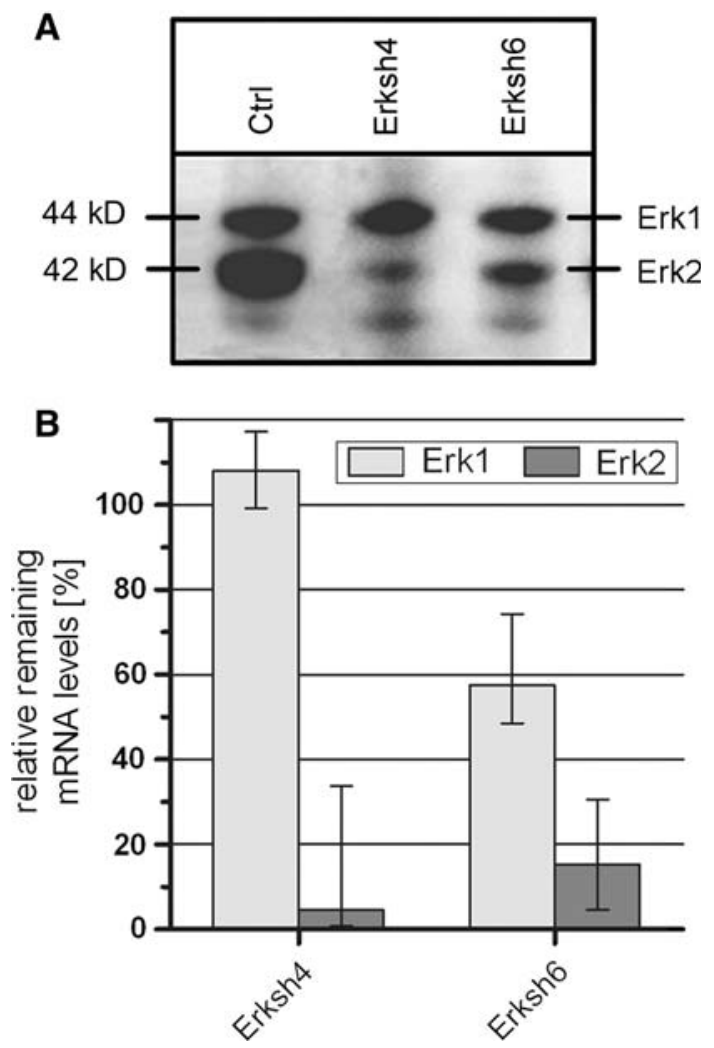

Fig. 2 Western blot and qRT-PCR analysis of downregulation efficiency of shRNA vectors. a ShRNA vectors were tested in ES cells, as described in section "Materials and Methods"; Western blot analysis revealed that Erksh4 and Erksh6 downregulated specifically ERK2, as compared to control samples ("Ctrl"). b Erksh4 and Erksh6 were further analyzed with qRT-PCR; the results confirmed the specific and significant reduction in ERK2 expression for Erksh4, while Erksh6 reduced ERK2, but affected also Erk1 mRNA level, and was therefore excluded from further experiments. Error bars show minimal and maximal results from triplicate measurements
Fig. 3 Tropism and downregulation efficiency of Erksh4 in vivo. a Schematic drawing of the amygdala at approximately $-1.82 \mathrm{~mm}$ from bregma (according to (Franklin and Paxinos, 1997)). b, c Tropism of the virus. Representative photomicrographs of a portion of the LA (blue square in (a)) which show the site of injection, immunostained for YFP (green) and NeuN (red) (b) and for YFP (green) and S100 $\beta$ (red) (c); ounterstaining with DAPI (blue) shows the single-cell nuclei. $\left(\mathbf{d}^{\prime}, \mathbf{d}^{\prime \prime}, \mathbf{d}^{\prime \prime \prime}\right)$ Higher magnification of labeled cells (white square (D) in (b)) to show the single signals for $\mathrm{NeuN}\left(\mathbf{d}^{\prime}\right)$ and venus $\left(\mathbf{d}^{\prime \prime}\right)$ and the yellow color of their "merge" $\left(\mathrm{D}^{\prime \prime \prime}\right)$ which highlights their co-localization; $\left(\mathbf{e}^{\prime}, \mathbf{e}^{\prime \prime}, \mathbf{e}^{\prime \prime \prime}\right)$ higher magnification of labeled cells (white square $(\mathrm{E})$ in $(\mathbf{c})$ ) to show the single signals for S100 $\beta\left(\mathbf{e}^{\prime}\right)$ and venus $\left(\mathbf{e}^{\prime \prime}\right)$ : in contrast with $\left(\mathbf{d}^{\prime \prime \prime}\right)$, their "merge" $\left(\mathbf{e}^{\prime \prime \prime}\right)$ shows only a faint co-localization of the two signals $(\mathbf{f}, \mathbf{g}, \mathbf{h}, \mathbf{i})$. Downregulation of Erk2 mRNA. Higher magnification of labeled cells showing the specificity of downregulation in $\mathrm{YFP}^{+}$cells infected with the Erksh4 virus $(\mathbf{h}, \mathbf{i})$ in comparison to $\mathrm{YFP}^{+}$cells infected with the control virus $(\mathbf{f}, \mathbf{g})$ : in $(\mathbf{f}, \mathbf{g})$ a clear ISH signal for Erk2 mRNA is visible in control infected cells (arrow heads), as high as in YFP $^{-}$ neighboring cells (asterisks); while in (h, i) the ISH signal is absent from $\mathrm{YFP}^{+}$cells (arrows), although still present in neighboring $\mathrm{YFP}^{-}$ cells (squares). $\mathbf{k}$ Double positive cells for YFP and Erk2 mRNA were counted in randomly selected sections from the amygdala of the four injected animals ( $n=2$ for each virus, bilaterally injected). The number of $\mathrm{YFP}^{+}, \mathrm{Erk}^{+}$cells was compared to the number of only $\mathrm{YFP}^{+}$cells. As shown in the figure, in the control injected animals (LacZsh), the relative number of Erk2 ${ }^{+}$cells was not significantly different from the only $\mathrm{YFP}^{+}$, while in the Erksh4 injected brains, this number was significantly reduced (ANOVA; ** $P \leq 0.01$ ), indicating an efficient downregulation of Erk2 also in vivo. Scale bars $=125 \mu \mathrm{m}$ in $(\mathbf{b}, \mathbf{c}) ; 60 \mu \mathrm{m}$ in D-d"' and E-e"'; $10 \mu \mathrm{m}$ in (f, $\mathbf{g}, \mathbf{h}, \mathbf{i})$

poly-L-lysine-coated slides, covered with anti-fading AquaPolyMount (Polysciences) and coverslips, and dried before confocal microscopy analysis.

\section{Microscopy Data Analysis}

The analysis was performed using a confocal microscope (Olympus, equipped with the FluoView1000 Software), scoring through amygdala's sections, from anterior to posterior levels, on both left and right sides, in at least two brains for each injected viral vector.

\section{Results}

Development and in vitro Test of Erk-Specific shRNA Vectors

Short hairpins targeting Erk1/2 or Erk2 alone were cloned into an expression vector under the control of the human U6 promoter (Fig. 1a, b). These vectors were electroporated into embryonic stem (ES) cells to test their gene silencing efficiency in vitro. Two days after transfection cells were lysed and the levels of ERK1 and ERK2 proteins were analyzed by western blotting of protein 

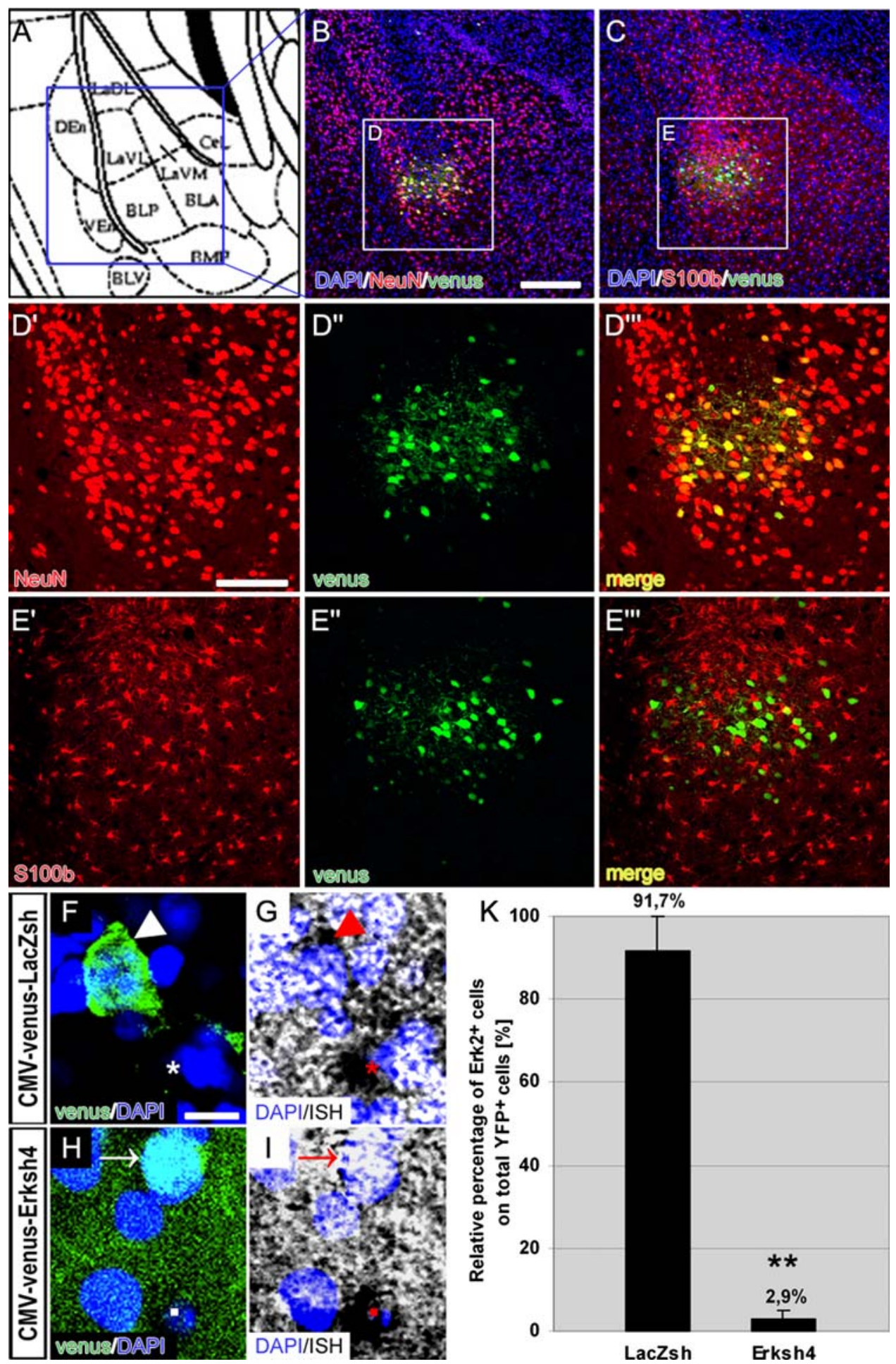

extracts. As shown in Fig. 2, both Erksh4 and Erksh6 did reduce ERK2 levels, but with different efficiencies. Notably, Erksh6 also seemed to reduce partially ERK1 activity (Fig. 2a, b). Therefore, we further analyzed the downregulation efficiency of both Erksh4 and Erksh6 via quantitative RT-PCR. As shown in Fig. 2b, Erksh4 almost completely inactivated Erk2 (4.6\% remaining mRNA level), leaving Erk1 unaffected. In contrast, the Erksh6 vector also reduced Erk1 mRNA (57.6\% remaining mRNA level). As we were interested in the specific inactivation of Erk2, the Erksh4 cassette was further used for in vivo experiments. 


\section{Performance of Viral shRNA Vectors in vivo}

To generate a viral shRNA expression vector the CMVVenus-U6Erksh4 expression unit was cloned into the backbone of AAV2 (Fig. 1c). As control, a shRNA targeting the mRNA coding for $\beta$-galactosidase ( $L a c Z$ ) was used (LacZsh). As shown in Fig. 1c, a stuffer sequence separated the U6-shRNA cassette from a second cassette expressing the yellow fluorescent protein (YFP) Venus under control of the CMV promoter, to allow the identification of infected cells upon in vivo injections. These viral preparations were stereotaxically injected into the LA of adult mice. The AAV2 vectors showed a clear preferential tropism for neurons (labeled with the specific neuronal marker NeuN [21]) and not for glia cells (labeled with the specific glial marker $\mathrm{S} 100 \beta$ [22]), as shown by immunohistochemistry for either DAPI/NeuN/Venus (Fig. 3b) or DAPI/S100 $\beta /$ Venus (Fig. 3c); moreover, the cells infected with the CMV-Venus-U6Erksh4 virus $\left(\mathrm{YFP}^{+}\right.$, Fig. 3h, white arrow) showed the specific downregulation of Erk2 mRNA (Fig. 3i, red arrow), while those transduced with the control CMV-Venus-U6LacZsh virus $\left(\mathrm{YFP}^{+}\right.$, Fig. 3f, white arrowhead), still showed Erk2 mRNA signals (Fig. 3g, red arrowhead). We quantified the proportion of double labeled cells $\left(\mathrm{YFP}^{+} ; E r k 2^{+}\right)$in comparison to the total number of infected cells $\left(\mathrm{YFP}^{+}\right)$cells for both injected viral vectors in randomly selected section areas from the LA. As shown in Fig. 3k, the relative number of Erk2 ${ }^{+}$cells in the control injected animals (LacZsh) was similar to the total number of only $\mathrm{YFP}^{+}$cells $(91.7 \pm 8.3 \%)$ while this number was strongly reduced in the CMV-Venus-U6Erksh4 injected animals (Erksh4, $2.9 \pm 2.2 \%)$. A one-way ANOVA analysis of the results confirmed a significant difference between the two groups $\left(F_{(1)}=13.38\right.$; ** $\left.P \leq 0.01\right)$. These in vivo results confirmed those obtained in vitro with the qRT-PCR (Fig. 2c) which showed the high efficiency of this shRNA in silencing Erk2 mRNA.

\section{Discussion}

In this study we identified shRNAs that efficiently knockdown the expression of either ERK1/2 simultaneously or only ERK2 in vitro (Fig. 2a, b). By using AAV as delivering agent we also tested the Erk2-specific shRNA in vivo and showed a concomitance between the downregulation efficiency in vivo with that obtained in vitro (Fig. 3). Therefore, our shRNAs can be used as tool for the further in vivo analysis of MAP kinase signaling in the etiopathogenesis of neuropsychiatric diseases. In particular, we found a shRNA, Erksh4, highly efficient for the silencing of Erk2 expression both in vitro and in vivo, that will prove useful to further analyze the role of this gene in specific regions of the adult brain (Figs. 2 and 3). Furthermore, we found a shRNA that targets and downregulates both Erk1 and Erk2 at the same time, but with different relative efficiency (Erksh6, Fig. 2a, b); this allows the functional analysis of both genes that are coexpressed in most regions of the adult brain and may exhibit functional redundancy [6]. With this shRNA we can compensate for the lack of pharmacological agents that inhibit ERK kinase activity and the inconvenience to inactivate both Erk genes by gene knockout technology. The differential relative downregulation efficiency makes it a useful tool for unmasking dose-dependent effects eventually related to only partial inactivation of the two genes' functions. With this shRNA it is possible to overcome the limitation of knockout alleles that allow only for $50 \%$ or $100 \%$ gene inactivation in the heterozygote or homozygote knockout configuration, respectively. In this study we show that AAV-mediated RNAi offers several advantages: (i) it can target molecules which are not amenable for pharmacological compounds; (ii) it targets preferentially neurons (Fig. 3b, $\mathrm{d}^{\prime \prime \prime}$ ), (iii) it allows the in vivo dissection of gene function in a restricted regional and temporal manner. These properties make it an excellent tool to unravel the specific neuronal role of Erk2 in complex behaviors. Nevertheless, to establish the utility of this system for behavioral analysis, additional experiments would be needed. As compared to other viral vectors that do not show neuronal tropism, e.g. lentivirus [23], the AAV approach offers specific cell-type selectivity. Since also glia cells can participate in the regulation of synaptic plasticity [24] it is important to validate the tropism of a viral agent that triggers RNAi. Our results of the AAV-CMV-VenusU6shRNA injections showed a clear concomitance between gene silencing in vitro and in vivo (compare Fig. 2 with Fig. 3). It is likely that the same concomitance applies to the other shRNA Erksh6, overcoming limitations of other methodologies for gradual and simultaneous gene inhibition. To our knowledge, Erk2 has been efficiently downregulated only in vitro via lentivirus-mediated RNAi [25]. Moreover, as recently shown by [26], there is an evident limitation not only in the availability of pharmacological compounds which target specifically single molecules like the ERKs, but also in the development of such inhibitors to analyze the function(s) of Ser/Thr kinases as ERKs. In this context, our study is relevant in demonstrating that the Erk2-specific AAV vector AAVCMV-Venus-U6Erksh4 provides a useful reagent for the silencing of Erk2 in vivo, enabling to establish an alternative tool to study anxiety and other neuropsychiatric disorders. 
Acknowledgements The authors are thankful to C. Birke and A. Kurz-Drexler for excellent technical assistance and to Cenix Biosciences for the performance of the qRT-PCR experiments. This work has been funded by the Volkswagen Foundation (to W.W.) and the Federal Ministry of Education and Research (BMBF) in the framework of the National Genome Research Network (to R.K.) (Förderkennzeichen 01GR0404). The authors are responsible for the contents of this publication. BDB performed the in vivo experiments, participated in the design of the study and wrote the manuscript. BW performed the in vitro experiments, participated in the design of the study and in writing the manuscript. WW supervised the work. RK participated in the design and coordination of the study and in writing the manuscript. All authors read and approved the final manuscript.

\section{References}

1. Bogoyevitch, M. A., \& Court, N. W. (2004). Counting on mitogen-activated protein kinases-ERKs 3, 4, 5, 6, 7 and 8. Cellular Signalling, 16, 1345-1354. doi:10.1016/j.cellsig.2004.05.004.

2. Schafe, G. E., Nadel, N. V., Sullivan, G. M., Harris, A., \& LeDoux, J. E. (1999). Memory consolidation for contextual and auditory fear conditioning is dependent on protein synthesis, PKA, and MAP kinase. Learning \& Memory (Cold Spring Harbor, N.Y.), 6, 97-110.

3. Grillon, C. (2002). Startle reactivity and anxiety disorders: Aversive conditioning, context, and neurobiology. Biological Psychiatry, 52, 958-975. doi:10.1016/S0006-3223(02)01665-7.

4. Selcher, J. C., Nekrasova, T., Paylor, R., Landreth, G. E., \& Sweatt, J. D. (2001). Mice lacking the ERK1 isoform of MAP kinase are unimpaired in emotional learning. Learning \& Memory (Cold Spring Harbor, N.Y.), 8, 11-19. doi:10.1101/lm.37001.

5. Satoh, Y., Endo, S., Ikeda, T., Yamada, K., Ito, M., Kuroki, M., et al. (2007). Extracellular signal-regulated kinase 2 (ERK2) knockdown mice show deficits in long-term memory; ERK2 has a specific function in learning and memory. The Journal of Neuroscience, 27, 10765-10776. doi:10.1523/JNEUROSCI.011707.2007

6. Di Benedetto, B., Hitz, C., Holter, S. M., Kuhn, R., Vogt Weisenhorn, D. M., \& Wurst, W. (2007). Differential mRNA distribution of components of the ERK/MAPK signalling cascade in the adult mouse brain. The Journal of Comparative Neurology, 500, 542-556. doi:10.1002/cne.21186.

7. Hannon, G. J. (2002). RNA interference. Nature, 418, 244-251. doi:10.1038/418244a.

8. Soutschek, J., Akinc, A., Bramlage, B., Charisse, K., Constien, R., Donoghue, M., et al. (2004). Therapeutic silencing of an endogenous gene by systemic administration of modified siRNAs. Nature, 432, 173-178. doi:10.1038/nature03121.

9. Gou, D., Weng, T., Wang, Y., Wang, Z., Zhang, H., Gao, L., et al. (2007). A novel approach for the construction of multiple shRNA expression vectors. The Journal of Gene Medicine, 9, 751-763. doi:10.1002/jgm.1080.

10. Aronoff, R., \& Petersen, C. C. (2006). Controlled and localized genetic manipulation in the brain. Journal of Cellular and Molecular Medicine, 10, 333-352. doi:10.1111/j.1582-4934. 2006.tb00403.x.

11. Davidson, B. L., Stein, C. S., Heth, J. A., Martins, I., Kotin, R. M., Derksen, T. A., et al. (2000). Recombinant adeno-associated virus type 2, 4, and 5 vectors: Transduction of variant cell types and regions in the mammalian central nervous system.
Proceedings of the National Academy of Sciences of the United States of America, 97, 3428-3432. doi:10.1073/pnas.050581197.

12. Xiao, X., Li, J., McCown, T. J., \& Samulski, R. J. (1997). Gene transfer by adeno-associated virus vectors into the central nervous system. Experimental Neurology, 144, 113-124. doi:10.1006/ exnr.1996.6396.

13. Taymans, J. M., Vandenberghe, L. H., Haute, C. V., Thiry, I., Deroose, C. M., Mortelmans, L., et al. (2007). Comparative analysis of adeno-associated viral vector serotypes $1,2,5,7$, and 8 in mouse brain. Human Gene Therapy, 18, 195-206. doi: 10.1089/hum.2006.178.

14. Kaplitt, M. G., Leone, P., Samulski, R. J., Xiao, X., Pfaff, D. W., O’Malley, K. L., et al. (1994). Long-term gene expression and phenotypic correction using adeno-associated virus vectors in the mammalian brain. Nature Genetics, 8, 148-154. doi:10.1038/ ng1094-148.

15. McCarty, D. M., Young, S. M., Jr, \& Samulski, R. J. (2004). Integration of adeno-associated virus (AAV) and recombinant AAV vectors. Annual Review of Genetics, 38, 819-845. doi: 10.1146/annurev.genet.37.110801.143717.

16. Paddison, P. J., Cleary, M., Silva, J. M., Chang, K., Sheth, N., Sachidanandam, R., et al. (2004). Cloning of short hairpin RNAs for gene knockdown in mammalian cells. Nature Methods, 1, 163-167. doi:10.1038/nmeth1104-163.

17. Hitz, C., Wurst, W., \& Kuhn, R. (2007). Conditional brain-specific knockdown of MAPK using Cre/loxP regulated RNA interference. Nucleic Acids Research, 35, e90. doi:10.1093/nar/ gkm475.

18. Rekas, A., Alattia, J. R., Nagai, T., Miyawaki, A., \& Ikura, M. (2002). Crystal structure of venus, a yellow fluorescent protein with improved maturation and reduced environmental sensitivity. The Journal of Biological Chemistry, 277, 50573-50578. doi: 10.1074/jbc.M209524200.

19. Auricchio, A., Hildinger, M., O'Connor, E., Gao, G. P., \& Wilson, J. M. (2001). Isolation of highly infectious and pure adenoassociated virus type 2 vectors with a single-step gravity-flow column. Human Gene Therapy, 12, 71-76. doi:10.1089/10430 3401450988.

20. Franklin, K. B. J., \& Paxinos, G. (1997). The mouse brain in stereotaxic coordinates. San Diego, CA, USA: Academic Press.

21. Mullen, R. J., Buck, C. R., \& Smith, A. M. (1992). NeuN, a neuronal specific nuclear protein in vertebrates. Development, 116, 201-211.

22. Nishiyama, H., Knopfel, T., Endo, S., \& Itohara, S. (2002). Glial protein S100B modulates long-term neuronal synaptic plasticity. Proceedings of the National Academy of Sciences of the United States of America, 99, 4037-4042. doi:10.1073/pnas.052020999.

23. Yee, J. K., Friedmann, T., \& Burns, J. C. (1994). Generation of high-titer pseudotyped retroviral vectors with very broad host range. Methods in Cell Biology, 43 Pt A, 99-112.

24. Seifert, G., Schilling, K., \& Steinhauser, C. (2006). Astrocyte dysfunction in neurological disorders: A molecular perspective. Nature Reviews. Neuroscience, 7, 194-206. doi:10.1038/nrn1870.

25. Vantaggiato, C., Formentini, I., Bondanza, A., Bonini, C., Naldini, L., \& Brambilla, R. (2006). ERK1 and ERK2 mitogenactivated protein kinases affect Ras-dependent cell signaling differentially. Journal of Biology (Online), 5, 14.

26. Fedorov, O., Marsden, B., Pogacic, V., Rellos, P., Muller, S., Bullock, A. N., et al. (2007). A systematic interaction map of validated kinase inhibitors with Ser/Thr kinases. Proceedings of the National Academy of Sciences of the United States of America, 104, 20523-20528. doi:10.1073/pnas.0708800104. 